\title{
Generation of a femtosecond electron microbunch train from a photocathode using twofold Michelson interferometer
}

\author{
M. Shevelev, ${ }^{1,2, *}$ A. Aryshev, ${ }^{1,3}$ N. Terunuma, ${ }^{1,3}$ and J. Urakawa ${ }^{1}$ \\ ${ }^{1}$ KEK: High Energy Accelerator Research Organization, 1-1 Oho, Tsukuba, Ibaraki 305-0801, Japan \\ ${ }^{2}$ Tomsk Polytechnic University, Institute of Physics and Technology, \\ Lenin Avenue 30, Tomsk 634050, Russian Federation \\ ${ }^{3}$ SOKENDAI: The Graduate University for Advanced Studies, 1-1 Oho, Tsukuba, Ibaraki 305-0801, Japan
}

(Received 20 April 2017; published 4 October 2017)

\begin{abstract}
The interest in producing ultrashort electron bunches has risen sharply among scientists working on the design of high-gradient wakefield accelerators. One attractive approach generating electron bunches is to illuminate a photocathode with a train of femtosecond laser pulses. In this paper we describe the design and testing of a laser system for an rf gun based on a commercial titanium-sapphire laser technology. The technology allows the production of four femtosecond laser pulses with a continuously variable pulse delay. We also use the designed system to demonstrate the experimental generation of an electron microbunch train obtained by illuminating a cesium-telluride semiconductor photocathode. We use conventional diagnostics to characterize the electron microbunches produced and confirm that it may be possible to control the main parameter of an electron microbunch train.
\end{abstract}

DOI: 10.1103/PhysRevAccelBeams.20.103401

\section{INTRODUCTION}

Electron bunches of picosecond-to-femtosecond duration for application in accelerator physics have been of great interest over the past decade. Ultrashort electron bunches can be used for applications in free-electron lasers $[1,2]$, wakefield acceleration [3,4] or coherent emission in the THz-frequency range [5-8]. Many methods for the generation of microbunch trains have been recently proposed and implemented. The most developed approaches include the bunch compression techniques [7,9-11], the formation of a microbunch train with a picosecond separation using an emittance exchanger combined with transverse beam masking [3,12] and a two-step approach based on self-wake energy modulation followed by a compression in chicane $[4,6,13]$. Yet all three of these techniques require significant beam line space allocation, a modification that would pose an inadmissible problem for a compact accelerator. A similar train of microbunches can be achieved by irradiating the photocathode of an $\mathrm{rf}$ gun with a train of femtosecond laser pulses.

The commonly applied technique to produce temporally shaped laser pulses relies on the use of birefringent crystals $[7,14]$. This method is quite simple: a single short polarized laser pulse entering a birefringent crystal splits in two pulses, each traveling at a different group velocity related to

*mvshev@icloud.com

Published by the American Physical Society under the terms of the Creative Commons Attribution 4.0 International license. Further distribution of this work must maintain attribution to the author(s) and the published article's title, journal citation, and DOI. the specific direction of the electric field. After a stack of $n$ crystals, one can generally obtain a sequence of $2^{n}$ pulse replicas. Regrettably, the approach considered would not permit any adjustment of the distance between laser pulses in the manner required by accelerator physics. The distance between the laser pulses can only be amended by changing the thickness of the birefringent crystal.

One alternative to the above-described approach using birefringent crystal would be a pulse-shaping technique using the phase and amplitude mask in the focal plane of a zero-dispersion pulse stretcher. This type of technique is usually employed to produce low-energy ultrafast pulse trains $[15,16]$. Damage to the mask residing in the focal plane of the stretcher hinders the use of such a method to produce high-energy trains. The number of pulses in a train and the distances between them are also determined by the design of the spatial mask. This technique cannot be exploited for a higher number of pulses.

Several optical schemes, variations of an interferometer, have appeared in the literature. The interference phenomena between two pulses can be harnessed to generate a laser pulse train with a variable number of pulses and pulse shapes [16-18]. The approach considered has a very strong condition for spectral chirp of initial laser pulses and does not allow the production of electron bunches of identical lengths with different distance between them. Another technique based on the Michelson interferometer (MI) uses multiple beam splitters and optical delay lines to produce a pulse train of $2^{k}$ pulses where $k$ is a positive integer [2,15,19,20]. Villa et al. [21] proposed a mixed scheme at most one birefringent crystal for each arm of the interferometer to arrange a trade-off between the flexibility 
of the delay lines and the ease of using the birefringent crystals. The most interesting application of a tunable narrow band $\mathrm{THz}$ source and high-gradient wakefield accelerator is a conventional MI scheme where the time delay between laser pulses in the train can be easily controlled. In this paper we report an experimental test using an MI to generate a laser pulse train for an rf gun in order to demonstrate the production of a train of four femtosecond electron microbunches.

The paper is divided into five sections. In Sec. II we describe modifications of the laser system for the rf gun, consider the interferometer design in detail, and characterize the temporal distribution of the laser pulse train for a photocathode rf gun. In Sec. III we consider and discuss an experimental facility to produce a femtosecond electron microbunch train. We then go on to consider and discuss the actual results obtained in Sec. IV. In Sec. V we present our conclusions and describe our future plans.

\section{LASER SYSTEM FOR GENERATING A FEMTOSECOND ELECTRON MICROBUNCH TRAIN}

The commercially available titanium-sapphire (Ti:Sa) laser technology was employed to produce ultrashort pulses. The ultrafast laser system makes use of a chirppulse amplification technique, followed by optical pulse compression [22], as illustrated in Fig. 1. The original scheme works as follows. Ultrashort (femtosecond) light pulses are generated by a Ti:Sa oscillator and chirped in time using a dispersive delay line consisting of a diffractiongrating arrangement to a picosecond level. Next, the pulses are amplified in a regenerative amplifier up to a few $\mu \mathrm{J}$, then further amplified with a multipass Ti:Sa amplifier to increase the laser pulse energy to an mJ level. After amplification, a second grating pair is used to compress the pulse back to a femtosecond duration.

As mentioned in Introduction, we applied the MI to produce a femtosecond laser pulse train. All currently proposed schemes [6,16-19] were designed for the $266 \mathrm{~nm}$ laser pulse. These designs are somewhat inconvenient for our purpose, as special optical components are required and the laser pulse duration can be expected to increase when the pulse goes through the MI components. Taking these factors into account, we decided to introduce the MI just after the regenerative amplifier (see Fig. 1), adopting a configuration that would allow the use of

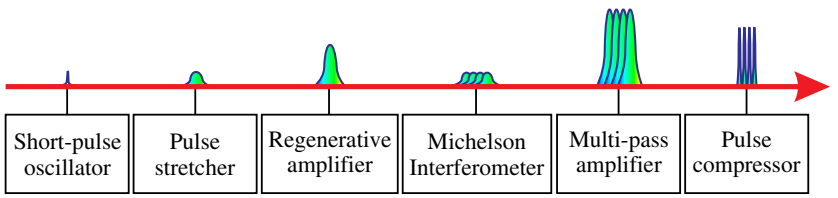

FIG. 1. Schematic diagram of a laser system based on chirppulse amplification. standard optical components and avoid the application of high-energy laser pulses.

Figure 2 shows the general layout of the MI. The device is a double-pass MI, where double passing is achieved with a polarizing beam splitter (PBS), quarter-wave plates and half-wave plates and prism reflectors. The first half-wave plate is employed to obtain incident light polarized in a plane at $45^{\circ}$ to the plane of incidence at the PBS $\left(R_{s}>99.5 \%, T_{p}>90 \%\right.$, where $R_{s}$ and $T_{p}$ are the reflectance for s-polarization component and the transmittance for p-polarization component, respectively) [23]. In the scheme adopted, the intensity of the reflected and transmitted pulses is approximately equal to that of the orthogonal planes of polarization. Each pulse then passes through a quarter-wave plate before being reflected normally onto one of the interferometer mirrors (the fixed mirror or the movable mirror No. 1). The retardation plates are oriented to rotate the planes of polarization of the pulses returning to the PBS by $90^{\circ}$ in two passes through the plates. The pulse that is first reflected is therefore now transmitted, and vice versa, so that both pulses now enter the retroreflection prism. When the retroreflected pulses return to the PBS they pass though the half-wave plate used to obtain light polarized in a plane at $45^{\circ}$ to the plane of incidence at the PBS. Thus, each pulse is then split into two pulse replicas. Two more passages through the quarter-wave plates restore the polarization condition of the pulses (the quarter waves before the fixed mirror and movable mirror No. 2). To rotate four orthogonal laser polarization states on the same angle before the multipass amplifier, the pulse train passes through the adjustable half-wave plate. It is used to rotate all polarization states with respect to the Ti:Sa amplified and compressed polarization direction and to balance or imbalance the pulse-by-pulse relative intensity within the microbunch train. Inconveniently, this decreases the efficiency of presented scheme by $50 \%$, as only the vertical polarization components are used at the pulse compressor. The energy chirp of laser pulses [24] prevents any undesirable interference phenomena between neighboring micropulses when time delay is more than $70 \mathrm{fs}$. The MI scheme eliminates any angle/position difference

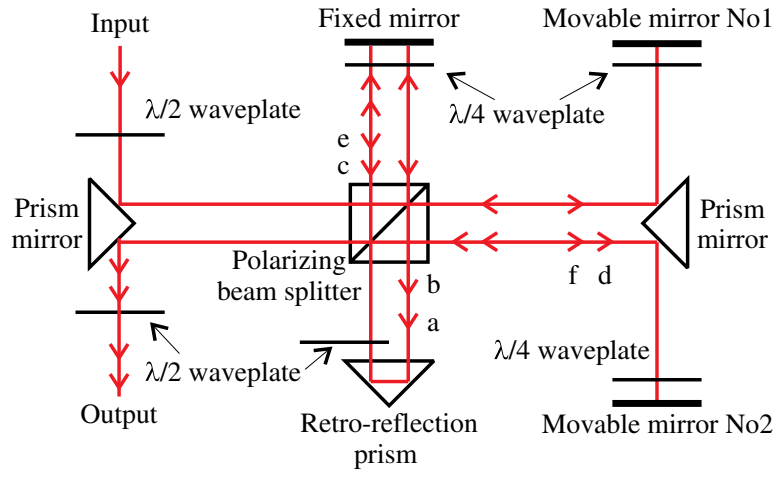

FIG. 2. Schematic of a method to produce four laser pulses. 
for the incoming and outgoing laser pulses after the regenerative amplifier and before the main multipass amplifier, respectively.

The presented system is more advanced for its ability to control the time delay and intensity ratio between micropulses than reported existing techniques. The blocking of one or two arms of the MI easily allows one to obtain the production of one or two micropulses that travel different passes. The linear motion of the movable interferometer mirrors results in a corresponding change in a time delay difference between the micropulses. The displacement of the first movable mirror causes a time delay between micropulses $a$ and $b$ (see Fig. 2). Meanwhile, the displacement of the second movable mirror simultaneously influences the time delay between micropulses in two pairs. The first pair consists of the $c$ and $e$; the second pair, of micropulses $d$ and $f$. The precision linear stages with a motorized actuator provide a $12 \mathrm{~mm}$ travel range with submicron resolution [23]. Note that the relative time offset of the system is proportional to 2 times the displacement of movable mirrors.

Next, the output pulses are amplified up to $2 \mathrm{~mJ}$ per micropulse by the multipass Ti:Sa amplifier and then recompressed back to $50 \mathrm{fs}$ (rms). As a result, $1.3 \mathrm{~mJ}$ from each micropulse at Ti:Sa fundamental harmonic $(\lambda=800 \mathrm{~nm})$ is available at the laser system output.
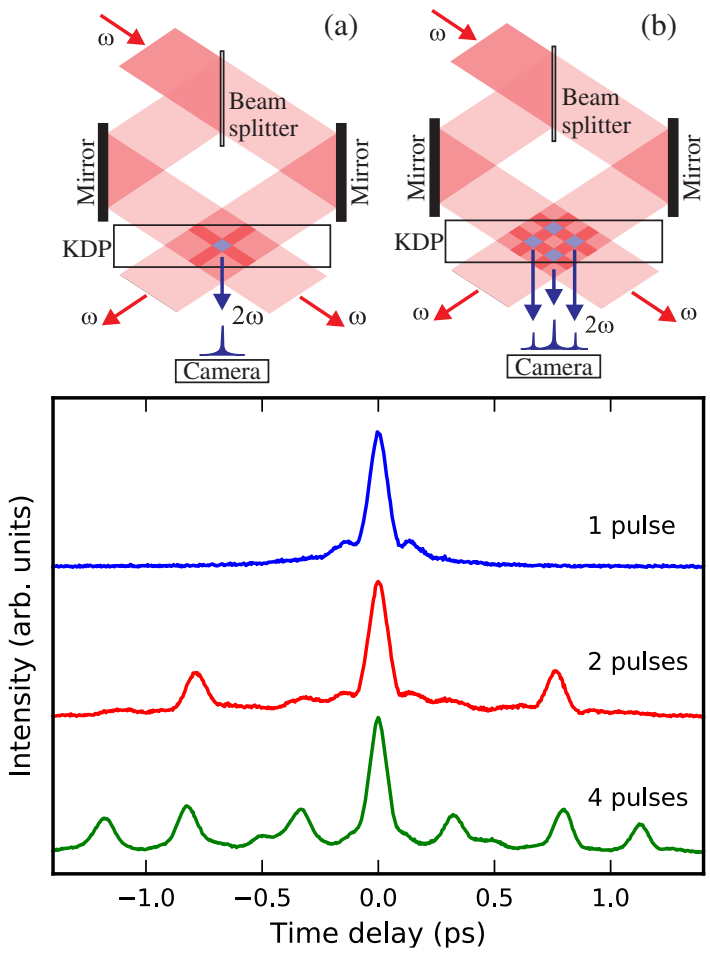

FIG. 3. Top row: Schematic of a femtosecond single-shot autocorrelator for the cases where (a) one and (b) two laser pulses interact in a potassium dihydrogen phosphate (KDP) crystal. Bottom: The examples of measured autocorrelation dependencies.
A femtosecond single-short autocorrelator was used to measure the micropulse duration and control time delay between the micropulses. The autocorrelator was designed to monitor the pulse width of an ultrafast laser system in the time range of 10 to $2000 \mathrm{fs}$ and spectral range of $720-880 \mathrm{~nm}$. The method used is based on the registration of the cross distribution of second harmonic energy produced in a potassium dihydrogen phosphate (KDP) crystal through nonlinear interaction of two beams with determined apertures. Figure 3 illustrates the gist of the technique for a simplified case of an initial laser pulse with a rectangular time and uniform-intensity cross distribution. Figure 3(b) shows an interaction of two micropulses in the KDP crystal. The additional micropulse causes three peaks to appear in the cross distribution of the second harmonic. The distance between the central and satellite peaks clearly corresponds to the time delay between micropulses. The autocorrelator thus allows to resolve the temporal distribution of the femtosecond pulses and measure the time delay between them in a single-shot mode. The bottom of Fig. 3 shows examples of autocorrelation dependence for different numbers of micropulses.

The proposed laser system scheme provides the generation up to four micropulses with adjustment of time delay between them and the energy balance in each micropulse pair.

\section{EXPERIMENTAL SETUP}

The experimental test was performed at the Laser Undulator Compact X-ray facility (LUCX) in the High Energy Accelerator Research Organization. Figure 4 shows a schematic diagram of the experimental setup. The LUCX is a multipurpose linear electron accelerator that was initially designed as an rf gun test bench and later extended for two projects: one to develop an x-ray source based on Compton scattering $[25,26]$ and one to investigate coherent radiation [27-31]. The LUCX consists of a high mode separation 3.6 cell rf gun with a $\mathrm{Cs}_{2} \mathrm{Te}$ photocathode [32] and a normal conductivity $1 \mathrm{~m} \mathrm{12}$-cell mode-separated linac booster [27]. Two klystrons are used to independently feed the rf gun and the accelerating structure. Diagnostics based on a ceramic luminescent screen was used to control the transverse density of an electron beam after the rf gun. The beam line downstream of the booster cavity includes

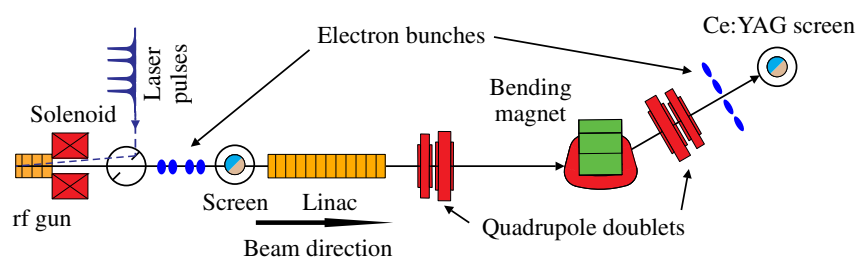

FIG. 4. Schematic layout of the LUCX facility showing the locations of the quadrupole magnets, bending magnet and diagnostic screens. 
TABLE I. Nominal operating settings for the rf gun, booster cavity and photocathode UV laser.

\begin{tabular}{ll}
\hline \hline Parameter & \multicolumn{1}{c}{ Value } \\
\hline Laser spot size on cathode & $\sim 480 \times 400 \mu \mathrm{m}^{2}$ \\
Laser rms pulse duration & $\sim 150 \mathrm{fs}$ \\
Bunch charge & $1-60 \mathrm{pC}$ \\
$E_{z}$ on cathode & $80 \mathrm{MV} / \mathrm{m}$ \\
rf gun phase & $20 \mathrm{rf} \mathrm{deg}$ \\
Peak $B_{z}$ (solenoid) & $0.225 \mathrm{~T}$ \\
Booster cavity gradient & $45 \mathrm{MV} / \mathrm{m}$ \\
\hline \hline
\end{tabular}

quadrupole doublets and a horizontal bending magnet that provides a spectrometer capability when combined with imaging of beam size at a $300 \mu$ m thick $0.5 \%$ cerium-doped yttrium aluminum garnet (Ce:YAG) screen. The horizontal dispersion value at the Ce:YAG screen location is $\left|\eta_{x}\right|=250 \mathrm{~mm}$. The nominal operating parameters of the LUCX are listed in Table I.

In the present study we irradiated the photocathode by a $266 \mathrm{~nm}$ femtosecond laser pulse from the third harmonic of the Ti:Sa femtosecond laser. We produced the desired femtosecond UV pulse using a collinear sum-frequencygeneration process of the third harmonic generation with two $\beta$-barium borate crystals. The maximum output pulse energy at $266 \mathrm{~nm}$ and the energy conversion efficiency of the femtosecond laser pulses from the fundamental to the third harmonic were $0.61 \mathrm{~mJ}$ and $3.8 \%$, respectively. After transport though a $\sim 11 \mathrm{~m}$ long optical beam line and passage through two vacuum windows and optical components, the estimated duration of the UV pulse is $\sim 150 \mathrm{fs}$ at the photocathode [33]. The transverse density of the UV pulse at the cathode was controlled via a virtual cathode consisting of a UV-sensitive CCD camera installed outside

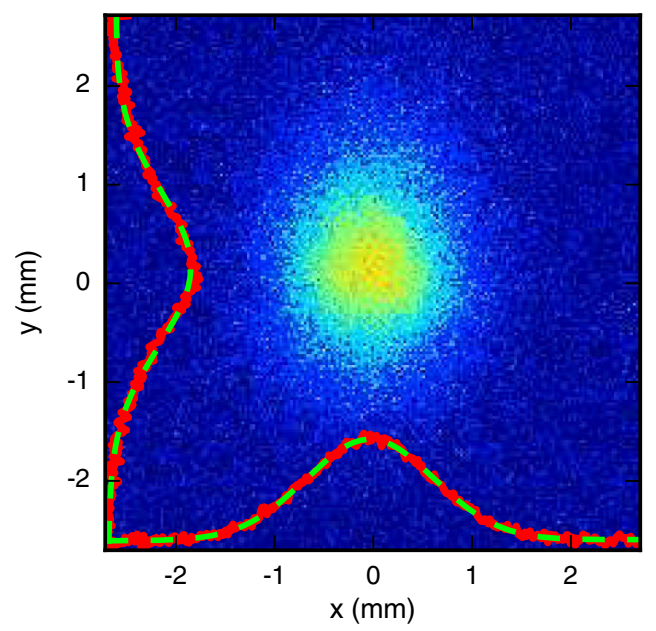

FIG. 5. Typical transverse spatial distribution of the UV laser pulse on the virtual photocathode with the associated projection (red solid traces) and the Gaussian fit functions (green dashed curves). of the vacuum chamber to provide a one-to-one optical image of the real cathode. Figure 5 shows the typical transverse spatial distribution of the UV laser pulse on the virtual cathode. The demonstrated transverse density of the UV pulse has root-mean-square (rms) dimensions of $\sim 480 \times 400 \mu \mathrm{m}^{2}$.

\section{MEASUREMENT RESULTS AND DISCUSSION}

We performed several experiments to demonstrate and characterize four microbunches in the same rf bucket. In this subsection we provide detailed descriptions of the experimental methods and results obtained. We also obtained simulated results that agreed well with the experimental results by performing simulations with ASTRA software [34] based on data from Table I.

We used the rf zero-phasing technique $[27,35]$ to confirm the generation of four microbunches in the train. In brief, an energy chirp is imparted on the bunches by operating the booster cavity off-crest. The microbunches in the train thus have different energies at the end of the booster cavity. When the electron bunches are dispersed in the spectrometer, the different time slices of the bunches are projected to different transverse position on the Ce:YAG screen. This technique is usually used for measuring the lengths of electron bunches. Its utility, however, hinges on a careful calibration and knowledge of the initial beam parameters [36]. We should also note that the single bunch length estimates obtained from measurement reflect a variation of the longitudinal phase-space ellipse caused by the rf gun beam dynamics.

An initial energy calibration was performed without energy chirp only for one microbunch with a charge of $1 \mathrm{pC}$. The averaged energy of the bunch was $E=$ $7.5 \pm 0.002 \mathrm{MeV}$ with an energy spread equal to $d E=12.0 \pm 0.5 \mathrm{keV}$, or $d E / E \sim 0.16 \%$. Figure 6 shows a typical transverse beam density for the different magnet current values and the measured calibration dependence. The rf zero-crossing point is determined by finding a phase that results in zero transverse image centroid movement in the spectrometer screen when the cavity is turned on and off at the same bending magnet setting.

We obtained the time calibration factor by measuring the correlation of the rf phase with the image centroid shift on the screen. The linear approximation of this correlation gives the scale of the horizontal image size in rf degrees, which can then be recalculated to the time scale as follows. The linear slope of the calibration is $0.11 \pm 5.44 \times 10^{-3} \mathrm{rf} \mathrm{deg} / \mathrm{mm}$, which is $106.99 \pm$ $5.29 \mathrm{fs} / \mathrm{mm}$ when one $\mathrm{rf}$ degree is assumed to be 972.6 fs. The experimental parameters in this measurement are $\lambda_{r f}$ of $10.5 \mathrm{~cm}$ (S-band $\mathrm{rf}, 2856 \mathrm{MHz}$ ) and linac rf amplitude $V_{r f}$ of about $45 \mathrm{MV}$. The estimated beam parameters in these measurements are an rms normalized 


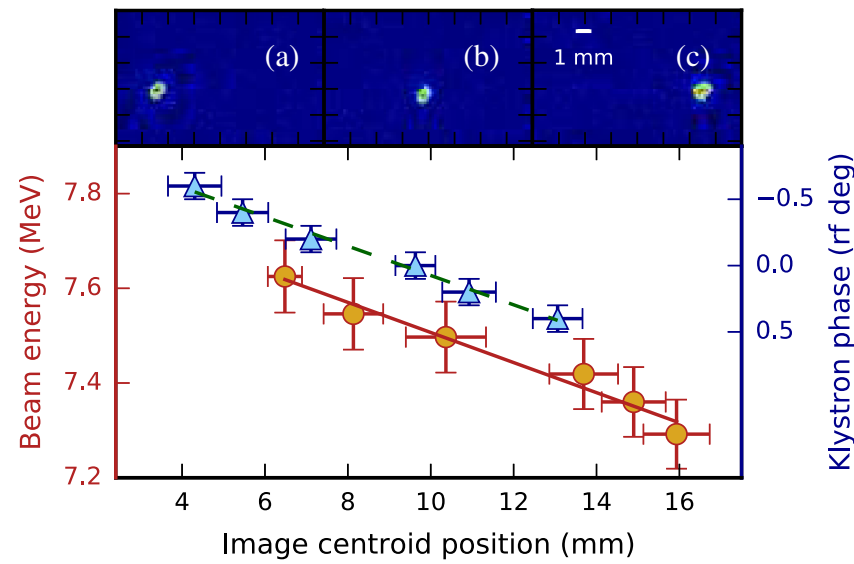

FIG. 6. Top row: The typical electron density distribution for the different magnet currents corresponds to: (a) $7.63 \mathrm{MeV}$, (b) $7.50 \mathrm{MeV}$, and (c) $7.30 \mathrm{MeV}$, respectively. Bottom: Beam image centroid position against electron beam energy (red dots) with a linear fit function (red solid curve): amplitude $7.82 \pm$ $0.03 \mathrm{MeV}$ and slope $3.18 \times 10^{-2} \pm 2.65 \times 10^{-3} \mathrm{MeV} / \mathrm{mm}$; beam image centroid against the rf phase of acceleration structure (blue triangles) with a linear fit function (green dashed curve): slope $0.11 \pm 5.44 \times 10^{-3} \mathrm{rf} \mathrm{deg} / \mathrm{mm}$.

transverse emittance $\epsilon_{x}$ of $0.3 \pi \mathrm{mm} \mathrm{mrad}$, rms longitudinal emittance of $0.15 \pi \mathrm{keVmm}$, and rms transverse beam size of $0.1 \times 0.1 \mathrm{~mm}^{2}$ at zero-phasing cavity. As the higherorder horizontal focusing terms caused by energy deviations assumed from beam line optics are 4 orders of magnitude smaller that the dispersion term, the maximum slope of the longitudinal phase-space ellipse $d E / d z$ is 4 times smaller than the rf slope $2 \pi q V_{r f} / \lambda_{r f}$ of $2.6 \mathrm{GeV} / \mathrm{m}$. The screen monitor is placed at a location where the dispersive beam size contributes more to the measured profile $\eta_{x} d E / E=400 \mu \mathrm{m}$ than the betatron size $\left(\beta_{x} \epsilon_{x} / \gamma\right)^{1 / 2}=200 \mu \mathrm{m}$, where $\gamma$ and $\beta_{x}$ are the Lorentz factor and the horizontal beta function, respectively. We disregard the Ce:YAG screen image-broadening effect, as the electron beam has a low charge. The rms resolution of approximately $30 \mathrm{fs}$ for this experimental arrangement is limited by the transverse beam size. Note, here, that the measured temporal electron distribution of the microbunches in the train obtained by this technique is valid not for the transverse profile measuring location, but for the zero-phasing cavity entrance. Further, the measured distribution linearly depends on the initial longitudinal electron distribution near the photocathode.

Measurements of the temporal distribution obtained on the Ce:YAG screen while the linac rf phase was set to the zero-crossing phase allowed us to confirm the generation of four microbunches in the train and determine the time separation between them. Figure 7 illustrates examples of distributions measured for different time delays between laser pulse pairs. The sequence of screen images shows the slow movement of one pair of bunches from left to right,

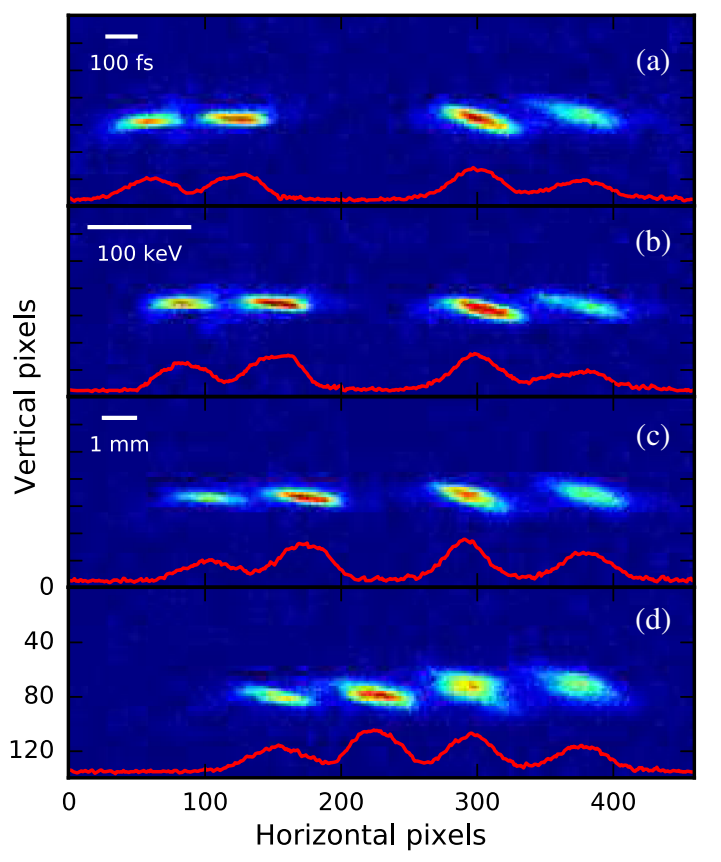

FIG. 7. Examples of the microbunch electron density distributions in a train measured on the Ce:YAG screen while the linac rf phase was set to zero-crossing phase. The acceleration phase of the rf gun is $20 \mathrm{rf}$ deg and time delay determined by movable mirror No. 2 is equal to 0.7 ps. Images (a), (b), (c) and (d) respectively correspond to the time delay between pairs of laser pulses equal to $1.20,1.10,0.88$ and $0.66 \mathrm{ps}$.

i.e. from low to high energy. Simultaneously the fundamental harmonic laser micropulse cross correlations were acquired to confirm laser micropulse spacing. The vertical projection on the screen and laser cross correlation were fitted by the Gaussian fit function, and the correlation of peak separation between the first (see the right bunch in Fig. 7) and third bunches was plotted against the time delay between pairs of laser pulses (see Fig. 8). One-hundred consecutive measurements were acquired for each data point. Note that the method used gives a similar scale factor of $123.86 \pm 16.26 \mathrm{fs} / \mathrm{mm}$ for the size of the horizontal image on the Ce:YAG screen, a value consistent with the phase calibration within standard deviations. The experimental conditions were numerically simulated with ASTRA tracking software to confirm the applicability of the rf zero-phasing technique to the microbunch train. As clearly seen in Fig. 8, the measurements and simulation results agree well.

The limitation of the developed scheme has been analyzed by Aryshev et al. [27]. Considering the case where two microbunches are generated, we demonstrated that the minimum spacing between bunches is determined by the space charge effect and the response time of the $\mathrm{Ce}_{2} \mathrm{Te}$ cathode, which is less than 370 fs. The next restriction, the maximum delay between bunches, is determined by the initial acceleration phase of the rf gun. Any 


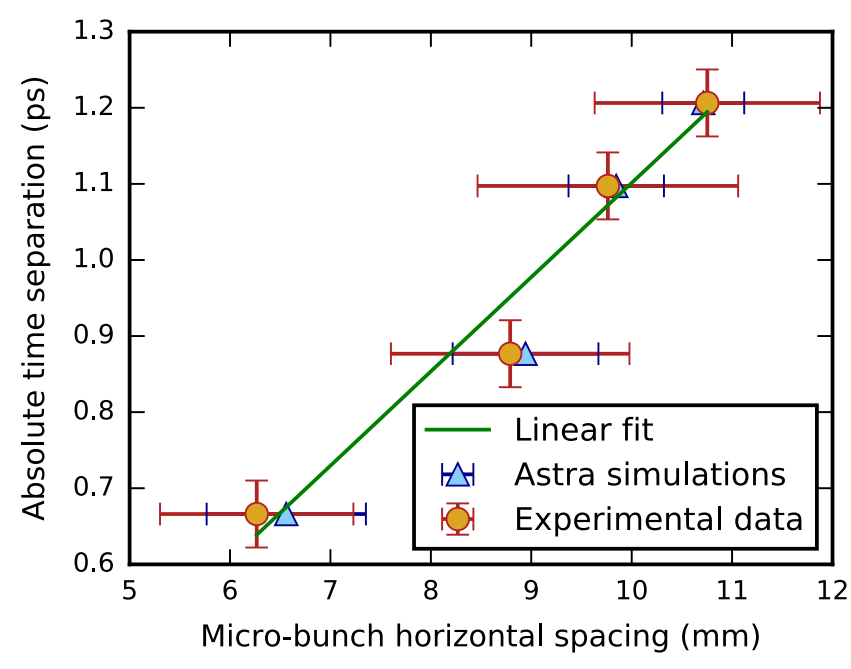

FIG. 8. Measured microbunch horizontal spacing between the electron pairs of bunches (red dots) as a function of the time delay between pairs of laser pulses with the linear fit function (green solid curve): slope $123.86 \pm 16.26 \mathrm{fs} / \mathrm{mm}$. The blue triangular dots correspond to simulations carried out with ASTRA.

variation of the time delay between laser pulses and acceleration phase of the rf gun will clearly lead to the acceleration of microbunches at different potentials, and thus to deviation of the bunch-to-bunch separation from its initial values. To more clearly illustrate the influence of the bunching velocity effect [37], we measured profiles of the electron density distribution in trains obtained on the Ce: YAG screen while varying the acceleration phases of the rf gun. Figure 9 shows that the distances between all of the microbunches simultaneously increase as the rf acceleration phase increases. On one hand, the bunching velocity

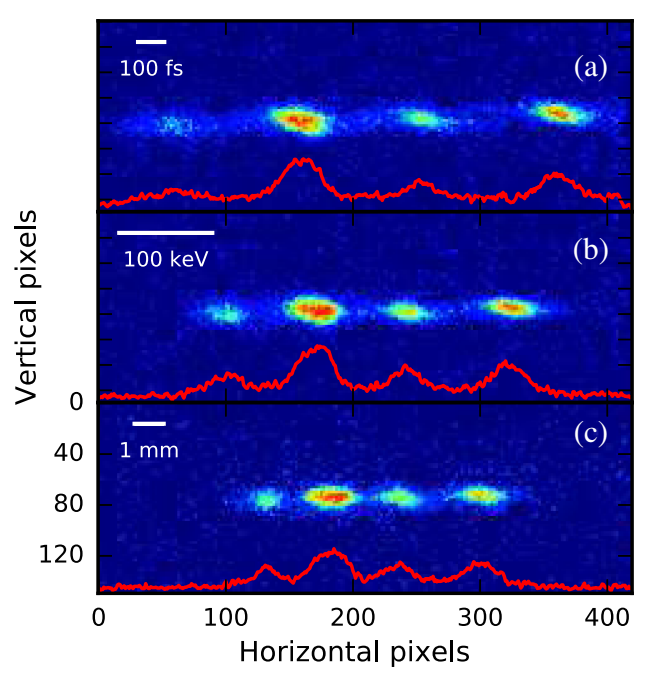

FIG. 9. Examples of the microbunch electron density distribution in trains measured on the Ce:YAG screen while varying the acceleration phases of the rf gun: (a) 25, (b) 15 and (c) $10 \mathrm{rf} \mathrm{deg}$. Note that time delays determined by movable mirrors No. 1 and No. 2 are 0.66 and 0.7 ps, respectively. effect begins to play a significant role when a large number of microbunches compose a train or when the time delay between microbunches slows to several picoseconds. On the other hand, the effect could be used as an additional tool for accurately adjusting the spacing between all microbunches.

As we mentioned briefly in Sec. II, the design of the double-pass MI allows us to control the power of laser pulses in pairs or between pairs and consequently the charge balance between microbunches in a train. Figure 10 gives examples of the measured temporal distribution obtained on the Ce:YAG screen. As the figure shows, the rotation position of a half-wave plate in the MI scheme allows us to suppress the charge of any pair in a train and/or microbunch in a pair. The control of the charge density in a microbunch train can be useful for compact wakefield accelerators in which the drive bunch should generate a wakefield while the following bunch harvests the energy from the wakefield and accelerating potential is achieved.

The scalability of the presented scheme towards higher electron microtrain charges is not limited from the point of the laser system as a sufficient energy margin is available. However, restrictions associated with the space-charge driven beam dynamics will affect resulting microbunch parameters at high charge operation [38]. As a possible overcome a higher acceleration gradient and bigger number of bunches per single accelerating rf cycle should be

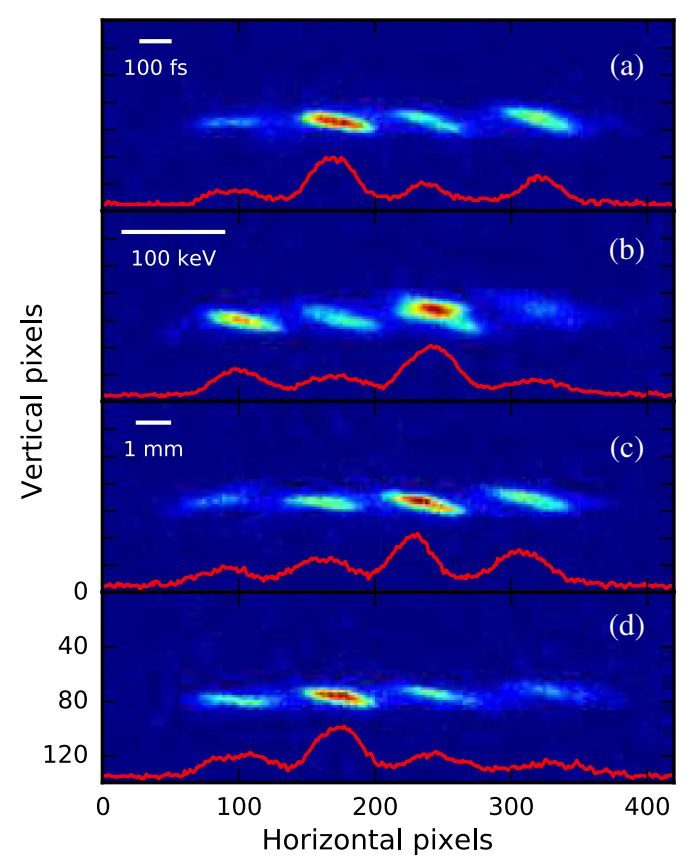

FIG. 10. Examples of the microbunch electron density distribution in trains measured on the Ce:YAG screen while varying the rotation position of the half-wave plates in MI. The acceleration phase of the rf gun is $20 \mathrm{rf}$ deg and time delays determined by movable mirrors No. 1 and No. 2 are 0.66 and 0.7 ps, respectively. 
considered. The presented MI scheme can be upscaled to a 4-, 6-, or even 8-times folded system able to generate up to 32 microbunches. The critical limitation on the number of microbunches accelerated in the same rf bucket will be determined by the length of microbunches and the time delays between them. In the case of a long microbunches train, the phase difference at the cathode may lead to unevenly separated pulses, different bunch lengths, energies and energy spreads after the rf gun. Also, the developed scheme has only two available delay lines instead of three. Thus, upscaling the system increases the missing degrees of freedom and consequently the upscaled system will be capable of generating equally separated pulses in each micropulse train. In this respect, for the plasma wakefield acceleration schemes, where the witness beam must have an additional about half plasma wavelength delay difference with respect to the driver bunches separation (usually one or an integer number of plasma wavelengths [39]) to shift from the decelerating to the accelerating phase of the wake, the missing degree of freedom may become a strong restriction. Nevertheless, the developed laser system is very useful for electron microbunch train generation, as it is more simple, compact and simply scalable than other existing schemes. By adding longer delays to the MI arms, the presented system can generate bunches in many subsequent $\mathrm{rf}$ buckets at the same rf phase, similar to the results presented in [40].

\section{SUMMARY}

In this study we applied twofold MI to produce four femtosecond laser pulses in order to create electron microbunches with variable delays on a photocathode. We demonstrated the generation of four femtosecond electron microbunches in one rf bucket using the rf zero-phasing technique. The scheme allowed us to control the delays between microbunches by means of mechanical delay and/or a bunching velocity effect. We then used half-wave plates to show that it may be possible to change the charge balance between microbunches in a train. To the best of our knowledge, this tuning level has not yet been demonstrated with four electron bunches. These features can be used for a number of applications and show good potential for development of a compact and narrow-band $\mathrm{THz}$ radiation source.

The KEK LUCX laser system will be further developed in the future to produce eight microbunches in a train. Experimental investigations of an undulator radiation and different types of coherent polarization radiation generated by a femtosecond comb beam are also foreseen.

\section{ACKNOWLEDGMENTS}

This work was supported by Photon and Quantum Basic Research Coordinated Development Program under the Ministry of Education, Culture, Sport, Science and
Technology, Japan, JSPS KAKENHI Grants No. 23226020 and No. 24654076, the Leverhulme Trust Network "Advanced Research on Generation of $\mathrm{THz}$ and X-ray Radiation" (IN-2015-012), the Federal Targeted Program of the Russian Federation (Agreement No. 14.578.21.0198 IDN RFMEFI57816X0198), and by the Competitiveness enhancement program of Tomsk Polytechnic University. The authors would like to thank S. Araki, Y. Honda, M. Fukuda, Y. Sumitomo, A. Konkov and other colleagues from the AGTaX collaboration for their valuable help, useful discussions and support. We acknowledge the extensive contribution of Dr. P. Karataev for the initial discussion on the twofold Michelson interferometer design.

[1] Y.-C. Huang, Desktop megawatt superradiant free-electron laser at terahertz frequency, Appl. Phys. Lett. 96, 231503 (2010).

[2] A. Marinelli, D. Ratner, A. A. Lutman, J. Turner, J. Welch, F.-J. Decker, H. Loos, C. Behrens, S. Gilevich, A. A. Miahnahri, S. Vetter, T. J. Maxwell, Y. Ding, R. Coffee, S. Wakatsuki, and Z. Huang, High-intensity double-pulse X-ray free-electron laser, Nat. Commun. 6, 6369 (2015).

[3] P. Muggli, V. Yakimenko, M. Babzien, E. Kallos, and K. P. Kusche, Generation of Trains of Electron Microbunches with Adjustable Subpicosecond Spacing, Phys. Rev. Lett. 101, 054801 (2008).

[4] S. Antipov, C. Jing, M. Fedurin, W. Gai, A. Kanareykin, K. Kusche, P. Schoessow, V. Yakimenko, and A. Zholents, Experimental Observation of Energy Modulation in Electron Beams Passing through Terahertz Dielectric Wakefield Structures, Phys. Rev. Lett. 108, 144801 (2012).

[5] P. Piot, Y.-E Sun, T. J. Maxwell, J. Ruan, A. H. Lumpkin, M. M. Rihaoui, and R. Thurman-Keup, Observation of coherently enhanced tunable narrow-band terahertz transition radiation from a relativistic subpicosecond electron bunch train, Appl. Phys. Lett. 98, 261501 (2011).

[6] S. Antipov, C. Jing, S. Baryshev, A. Kanareykin, D. Wang, W. Gai, A. Zholents, and M. Fedurin, THz radiation generation in a multimode wakefield structure, in Proceedings of 6th International Particle Accelerator Conference, Richmond, VA (JACoW, Richmond, 2015), p. 1929.

[7] E. Chiadroni, M. Bellaveglia, P. Clvani, M. Castellano, L. Catani, A. Cianchi, G. Di Pirro, M. Ferrario, G. Gatti, O. Limaj, S. Lupu, B. Marchetti, A. Mostacci, E. Pace, L. Palumbo, C. Ronsivalle, R. Pompili, and C. Vaccarezza, Characterization of the $\mathrm{THz}$ radiation source at the Frascati linear accelerator, Rev. Sci. Instrum. 84, 022703 (2013).

[8] D. Daranciang, J. Goodfellow, M. Fuchs, H. Wen, Sh. Ghimire, D. A. Reis, H. Loos, A. S. Fisher, and A. M. Lindenberg, Single-cycle terahertz pulses with $>0.2 \mathrm{~V} / \AA$ field amplitudes via coherent transition radiation, Appl. Phys. Lett. 99, 141117 (2011).

[9] I. Nozawa, K. Kan, J. Yang, A. Ogata, T. Kondoh, M. Gohdo, K. Norizawa, H. Kobayashi, H. Shibata, S. Gonda, and Y. Yoshida, Measurement of $<20$ fs bunch length using coherent transition radiation, Phys. Rev. ST Accel. Beams 17, 072803 (2014). 
[10] M. Ferrario et al., Experimental Demonstration of Emittance Compensation with Velocity Bunching, Phys. Rev. Lett. 104, 054801 (2010).

[11] P. Piot, L. Carr, W. S. Graves, and H. Loos, Subpicosecond compression by velocity bunching in a photoinjector, Phys. Rev. ST Accel. Beams 6, 033503 (2003).

[12] Y.-E Sun, P. Piot, A. Johnson, A. H. Lumpkin, T. J. Maxwell, J. Ruan, and R. Thurman-Keup, Tunable Subpicosecond Electron-Bunch-Train Generation Using a Transverse-To-Longitudinal Phase-Space Exchange Technique, Phys. Rev. Lett. 105, 234801 (2010).

[13] S. Antipov, M. Babzien, C. Jing, M. Fedurin, W. Gai, A. Kanareykin, K. Kusche, V. Yakimenko, and A. Zholents, Subpicosecond Bunch Train Production for a Tunable mJ Level THz Source, Phys. Rev. Lett. 111, 134802 (2013).

[14] L. Yan, Q. Du, Y. Du, J. Hua, W. Huang, and Ch. Tang, UV pulse shaping for the photocathode rf gun, Nucl. Instrum. Methods Phys. Res., Sect. A 637, S127 (2011).

[15] C. W. Siders, J. L. W. Siders, A. J. Taylor, S.-G. Park, and A. M. Weiner, Efficient high-energy pulse-train generation using a $2^{n}$-pulse Michelson interferometer, Appl. Opt. 37, 5302 (1998).

[16] M. Boscolo, M. Ferrario, I. Boscolo, F. Castelli, and S. Cialdi, Generation of short $\mathrm{THz}$ bunch trains in a $\mathrm{rf}$ photoinjector, Nucl. Instrum. Methods Phys. Res., Sect. A 577, 409 (2007).

[17] J. G. Neumann, R. B. Fiorito, P. G. O'Shea, H. Loos, B. Sheehy, Y. Shen, and Z. Wu, Terahertz laser modulation of electron beams, J. Appl. Phys. 105, 053304 (2009).

[18] Y. Shen, X. Yang, G. L. Carr, Y. Hidaka, J. B. Murphy, and $\mathrm{X}$. Wang, Tunable Few-Cycle and Multicycle Coherent Terahertz Radiation from Relativistic Electrons, Phys. Rev. Lett. 107, 204801 (2011).

[19] Sh. Liu and Y.-Ch. Huang, Generation of prebunched electron beams in photocathode rf gun for THz-FEL superradiation, Nucl. Instrum. Methods Phys. Res., Sect. A 637, S172 (2011).

[20] Yu. Ding, Z. Huang, and R. D. Ruth, Two-bunch selfseeding for narrow-bandwidth hard x-ray free-electron lasers, Phys. Rev. ST Accel. Beams 13, 060703 (2010).

[21] F. Villa, M. P. Anania, M. Bellaveglia, F. Bisesto, E. Chiadroni, A. Cianchi, A. Curcio, M. Galletti, D. Di Giovenale, G. Di Pirro, M. Ferrario, G. Gatti, M. Moreno, M. Petrarca, R. Pompili, and C. Vaccarezza, Laser pulse shaping for high gradient accelerators, Nucl. Instrum. Methods Phys. Res., Sect. A 829, 446 (2016).

[22] S. Backus, Ch. G. Durfee, M. M. Murnane, and H. C. Kaqpteyn, High power ultrafast lasers, Rev. Sci. Instrum. 69, 1207 (1998).

[23] http://www.thorlabs.co.jp.

[24] M. Born and E. Wolf, Principles of Optics, 4th ed. (Pergamon Press, New York, 1970).

[25] K. Sakaue, M. Washio, S. Araki, M. Fukuda, Y. Higashi, Y. Honda, T. Omori, T. Taniguchi, N. Terunuma, J. Urakawa, and N. Sasao, Observation of pulsed x-ray trains produced by laser-electron Compton scattering, Rev. Sci. Instrum. 80, 123304 (2009).

[26] M. Fukuda, S. Araki, A. Deshpande, Y. Higashi, Y. Honda, K. Sakaue, N. Sasao, T. Taniguchi, N. Terunuma, and
J. Urakawa, Upgrade of the accelerator for the laser undulator compact x-ray source (LUCX), Nucl. Instrum. Methods Phys. Res., Sect. A 637, S67 (2011).

[27] A. Aryshev, M. Shevelev, Y. Honda, N. Terunuma, and J. Urakawa, Femtosecond response time measurements of a $\mathrm{Cs}_{2}$ Te photocathode, Appl. Phys. Lett. 111, 033508 (2017).

[28] A. Aryshev, S. Araki, M. Fukuda, P. Karataev, A. Konkov, G. Naumenko, A. Potylitsyn, K. Sakaue, L. Sukhikh, N. Terunuma, D. Verigin, and J. Urakawa, Observation of the stimulated coherent diffraction radiation in an open resonator at LUCX facility, Nucl. Instrum. Methods Phys. Res., Sect. A 763, 424 (2014).

[29] M. Shevelev, A. Aryshev, S. Araki, M. Fukuda, P. Karataev, N. Terunuma, and J. Urakawa, Coherent radiation spectrum measurements at KEK LUCX facility, Nucl. Instrum. Methods Phys. Res., Sect. A 771, 126 (2015).

[30] A. Aryshev, A. Potylitsyn, G. Naumenko, M. Shevelev, K. Lekomtsev, L. Sukhikh, P. Karataev, Y. Honda, N. Terunuma, and J. Urakawa, Monochromaticity of coherent Smith-Purcell radiation from finite size grating, Phys. Rev. Accel. Beams 20, 024701 (2017).

[31] H. Harrison, G. Doucas, A. J. Lancaster, I. V. Konoplev, H. Zhang, A. Aryshev, M. Shevelev, N. Terunuma, and J. Urakawa, Novel approach to the elimination of background radiation in a single-shot longitudinal beam profile monitor, in Proceedings of 5th International Beam Instrumentation Conference (JACoW, Barcelona, 2016), p. 471.

[32] A. Deshpande, S. Araki, M. Fukuda, K. Sakaue, N. Terunuma, J. Urakawa, and M. Washio, Experimental results of an $\mathrm{rf}$ gun and the generation of a miltibunch beam, Phys. Rev. ST Accel. Beams 14, 063501 (2011).

[33] I. Walmsley, L. Waxer, and Ch. Dorrer, The role of dispersion in ultrafast optics, Rev. Sci. Instrum. 72, 1 (2001).

[34] K. Flöttmann, ASTRA: A space charge algorithm, User's Manual, available at http://www.desy.de.

[35] D. X. Wang, A. G. Krafft, and C. K. Sinclair, Measurement of femtosecond electron bunches using a rf zero-phasing method, Phys. Rev. E 57, 2283 (1998).

[36] J. Seeman, Beam dynamics verification in linacs of linear colliders, in Proceedings of Linear Accelerator Conference (JACoW, Williamsburg, 1988), p. 487.

[37] H. Iijima, M. Uesaka, F. Sakamoto, T. Ueda, N. Kumagai, and L. Serafini, Experimental verification of velocity bunching via shot-by-shot measurement at s-band photoinjector and linac, Jpn. J. Appl. Phys. 44, 5249 (2005).

[38] M. Shevelev, A. Aryshev, Y. Honda, N. Terunuma, and J. Urakawa, Influence of space charge effect in femtosecond electron bunch on coherent transition radiation spectrum, Nucl. Instrum. Methods Phys. Res., Sect. B 402, 134 (2017).

[39] P. Chen, J. M. Dawson, R. W. Huff, and T. Katsouleas, Acceleration of Electrons by the Interaction of a Bunched Electron Beam with a Plasma, Phys. Rev. Lett. 54, 693 (1985).

[40] R. A. Marsh, D. J. Gibson, and Y. Hwang, Initial performance measurements of multi-GHz electron bunch trains, in Proceedings of 8th International Particle Accelerator Conference (JACoW, Copenhagen, 2017), p. 795. 